\title{
Force fluctuations experienced by a boundary wall subjected to a granular flow in two distinct systems
}

\author{
François Kneib ${ }^{1, \star}$, Thierry Faug ${ }^{1, \star \star}$, Frédéric Dufour ${ }^{2}$, and Mohamed Naaim ${ }^{1}$ \\ ${ }^{1}$ Univ. Grenoble-Alpes, Irstea, ETGR, 38402 St-Martin d'Hères, France \\ ${ }^{2}$ Univ. Grenoble-Alpes, CNRS, 3SR, F-38000 Grenoble, France
}

\begin{abstract}
The present paper describes a numerical study of force fluctuations experienced by a boundary wall subjected to a granular flow on two distinct systems, namely a lid-driven cavity and an immersed wall system. Though the two systems exhibit different time-averaged dynamics, the force fluctuations experienced by the boundary wall show robust features in terms of the shapes of the probability density distributions and autocorrelation functions, under a wide range of boundary confinement pressure and shearing velocity imparted to the granular flow at the top of the system. This study identifies the key link between the grain-wall force fluctuations and the $\mu(I)-$ rheology while moving from quasistatic to inertial regime.
\end{abstract}

\section{Introduction}

The problem of an assembly of grains in interaction with a boundary wall is relevant for a number of real-world applications involving granular materials, such as the transport of particle in food processing or mining industry, the impact of large-scale avalanche-flows with civil engineering structures in geophysics, the design of technological earthmoving equipments used to work with the soils in agriculture, etc. Understanding the force experienced by a boundary wall in interaction with a granular flow is crucial but little is known about the fluctuating part of the granular force under slow to fast flow regimes. The distributions of grain-grain or grain-wall contact forces (noted $f$ ) in static or quasistatic systems received a great attention, as reported in $[1,2]$ and a number of references therein. A salient feature in static granular media is a relatively robust exponential decrease at large forces, while the distributions at small forces may show very different behaviours (absence or presence of a plateau-even a peak, non-zero probability at $f=0$ ), depending on many parameters associated with the shear history of the system. By contrast, only a few studies were devoted to force fluctuations at stake in flows of granular materials (see for instance [3]).

The present study investigates the distributions of grain-wall forces, as well as force time-series autocorrelation, when a granular flow interacts with a rigid boundary wall, under a wide range of macroscopic boundary shearing velocity $U$ and confinement pressure $P$ imparted to the granular bulk. The approach is based on the crosscomparison between two distinct granular flow systems simulated by discrete element method (DEM). The two

\footnotetext{
${ }^{\star}$ e-mail: francois.kneib@irstea.fr
}

${ }^{\star}$ e-mail: thierry.faug@irstea.fr systems and their time-averaged dynamics are briefly described in section 2. Section 3 is devoted to the grain-wall force distributions on the entire height of the wall and on portions of wall. The analysis of force time-series autocorrelation is addressed in section 4. Finally, a conclusion is given in section 5 .

\section{Two distinct granular systems studied}

\subsection{Flow-wall geometries and methods}

This section gives a brief summary of the two systems studied, both involving a boundary wall that is subjected to a planar and gravity-free granular flow. The first system, namely the granular lid-driven cavity, is a closed system for which the grains are forced to stay inside the cavity, thus producing a perpetual recirculation of the grains in the whole volume. The second system, namely the immersed wall granular system, is an opened system which enables the grains to escape by passing over an immersed wall, while other grains are trapped behind the wall-like obstacle. Both systems are simulated thanks to DEM, using the open-source YADE software [4].

The contact forces between the spherical particles of diameter $d$ were modelled using a damped linear spring law for the normal direction and a linear spring with a static Coulomb threshold law for the tangential direction. Four microscopic parameters were required for the simulations presented here: the normal stiffness $k_{n}$, the tangential stiffness $k_{t}$, the interparticle friction coefficient $\mu$ and the normal restitution coefficient $e$. All simulations were conducted in the limit of rigid grains, varying $k_{n}$ and $k_{t}$ accordingly, with respect to the macroscopic pressure imparted by the top wall to the system. We considered 
$\mu=0.5$ and $e=0.5$. Full information on how the values of those parameters were implemented is given in our initial study of the lid-driven cavity system [5].

For both systems, we defined the two dimensionless following parameters: $N_{U}=U / \sqrt{g d}$ and $N_{P}=$ $P /\left(\Phi \rho_{P} g H\right)$ where $H$ is the height of the system (equal to $30 d$ with $d=1 \mathrm{~mm}$ in the simulations presented here), and $\rho_{P}$ was the particle density taken equal to $2500 \mathrm{~kg}$ $\mathrm{m}^{-3}$ (corresponding to the density of glass beads generally used in small-scale laboratory experiments). The systems lengths $L$ were set to five times the height of grains: $L / H=5$. A polydispersity of $\pm 15 \%$ was given to the grains in order to avoid crystallization. Note that we considered typical pressures $\Phi \rho_{P} g H$ (where $\Phi$ was a typical volume fraction taken equal to 0.6 for a dense threedimensional packing) and typical velocities $\sqrt{g d}$ in order to define $N_{P}$ and $N_{U}$, though the systems simulated by DEM were gravity-free in the present study. This choice was made for convenience if laboratory experiments under gravity are developed in the future and compared to the DEM simulations presented in the paper. For both systems, $N_{U}$ and $N_{P}$ were typically varied in the ranges $[1 ; 20]$ and $[0.01 ; 100]$, respectively.

Based on the boundary conditions imposed at the top in terms of shearing velocity $U$ and confinement pressure $P$, a macroscopic inertial number can be defined as the ratio between the typical time $t_{P}=d \sqrt{\rho / P}$, associated with the top confinement pressure $P$, and the typical time $t_{U}=H / U$, equal to the inverse of the macroscopic shear rate over the height of the sample: $I_{M}=\frac{U}{H} d \sqrt{\rho / P}$.

\subsubsection{The granular lid-driven cavity}

The two-dimensional granular lid-driven cavity simulated by DEM consists of a planar assembly of spheres trapped into a box made of four walls, as sketched in Fig. 1. The perpetual recirculation of the grains within the cavity (closed system) produced streamlines showing the formation of a vortex in the whole volume of the cavity. The mean force on the right sidewall (see Fig. 1) was found to fit the empirical following scaling:

$$
\bar{F}=\left(r+\frac{\Delta r}{1+I_{M}^{0} / I_{M}}\right) P L d,
$$

where $r=0.53, \Delta r=0.33$ and $I_{M}^{0}=0.2$ in this study (see detail in [5, 6]). The values of $r$ and $\Delta r$ depend on the mechanical properties of the grains, for instance the interparticle friction, as reported in [6] where results from DEM simulations with $\mu=0.27$ are presented too.

\subsubsection{The immersed wall granular system}

The immersed wall granular system consists of a horizontal flow of spheres passing over a wall of height $h=20 d$, as sketched in Fig. 2. This system was simulated by DEM using periodic conditions. The immersed wall-like obstacle led to the formation of a dead zone behind the wall, this dead zone co-existing with an overlying sheared granular

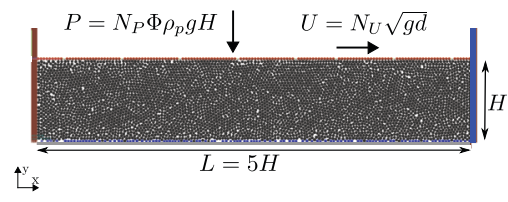

Figure 1. Geometry and boundary conditions for the granular lid-driven cavity simulated by DEM

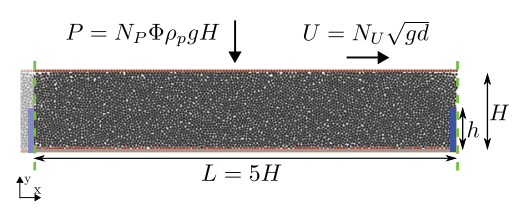

Figure 2. Geometry and boundary conditions for the immersed wall granular system simulated by DEM. Periodic conditions were used, thus explaining the presence of a wall on the left side (lighter-colored area), which is a duplicate of the wall (of interest in our study) on the right side

flow. As a result, the mean force on the wall was found to obey the empirical following relation:

$$
\bar{F}=\left(r^{*}+\frac{\Delta r^{*}}{1+I_{M}^{* 0} / I_{M}^{*}}\right) P h d,
$$

where $r^{*}=1.4, \Delta r^{*}=1.9$ and $I_{M}^{* 0}=0.3$ in the present study (see detail in [7]). The values of $r^{*}$ and $\Delta r^{*}$ depend on the mechanical properties of grains. $I_{M}^{*}$ is the macroscopic inertial number defined with the difference between the height of the system (the height of grains) and the immersed wall height: $I_{M}^{*}=\frac{U}{H-h} d \sqrt{\rho / P}$.

\subsection{Distinct time-averaged dynamics}

Because the two systems presented above are by construction different, they show two different time-averaged dynamics. First, a standing vortex-like structure is formed inside the cavity volume [5, 6], while longitudinal streamlines with a curvature in the vicinity of the wall take place in the immersed wall system [7]. Second, the mean force scalings allowed us to identify different relevant spatial scales specific to each system. The relevant pressure force is $P L d$ for the cavity (see Eq. (1)), while it is $P h d$ for the immersed wall system (see Eq. (2)). The relevant spatial scale for the macroscopic inertial number is the system height $H$ for the cavity (see Eq. (1)), while it is the height difference $H-h$, namely the height of the granular layer overflowing the wall, for the second system (see Eq. (2)). Much more details about the specific features of the liddriven cavity and the immersed wall system can be found in [5, 6] and [7], respectively.

However, we show in the following how these two distinct systems exhibit very similar - not to say, for some aspects, identical-key features in terms of the force distributions and the force time-series autocorrelations on the boundary wall, and we will identify a robust signature of the transition from quasistatic to dense inertial granular flow regime. 

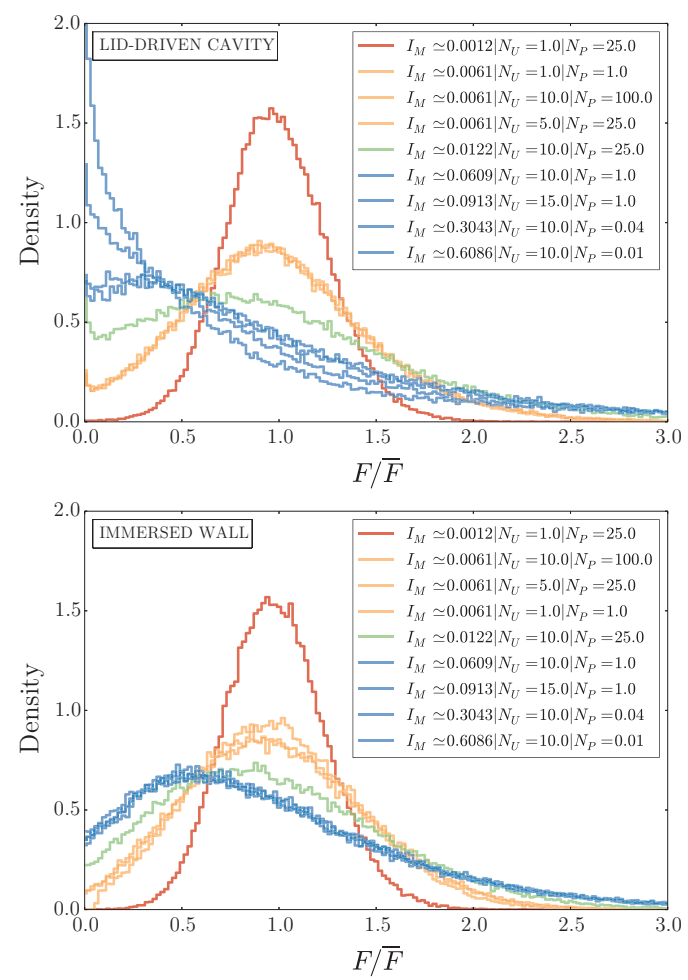

Figure 3. Distributions of total force experienced by the boundary wall for the lid-driven cavity (top panel) and the immersed wall system (bottom panel), for different values of $I_{M}$

\section{Grain-wall force distributions}

\subsection{At the scale of the entire wall}

Figure 3 shows the distributions of total force on the boundary wall for the lid-driven cavity (top panel) and the immersed wall system (bottom panel) for different values of $I_{M}$. First, it is worthwhile to note that the distributions are entirely controlled by the value of $I_{M}$, whatever the pair $(U, P)$ (see the curves for $I_{M}=6.110^{-3}$ obtained with three different pairs $(U, P)$ for the two systems). Second, it is found that they generally evolve from an exponentiallike form at high forces (much larger than the mean) with the presence of a plateau/peak at small forces for high $I_{M}$, toward a Gaussian-like form at the lowest $I_{M}$. We observe a difference between the cavity and the immersed wall at the highest $I_{M}$ : the cavity exhibit purely exponential shapes without any peak, while the peak at small forces is still present in the immersed wall system.

\subsection{At the scale of a portion of wall}

For both systems and a given $I_{M}$, our simulations showed that the distribution of the force $F_{i}$ on each portion $i$ of the boundary wall was independent of the vertical location along the boundary wall, excluding the extreme locations at the top and the foot of the wall (not shown here: details can be found in [6] for the cavity and [7] for the immersed wall). As a result, we could average the distributions and extract robust mean values of distributions as functions of
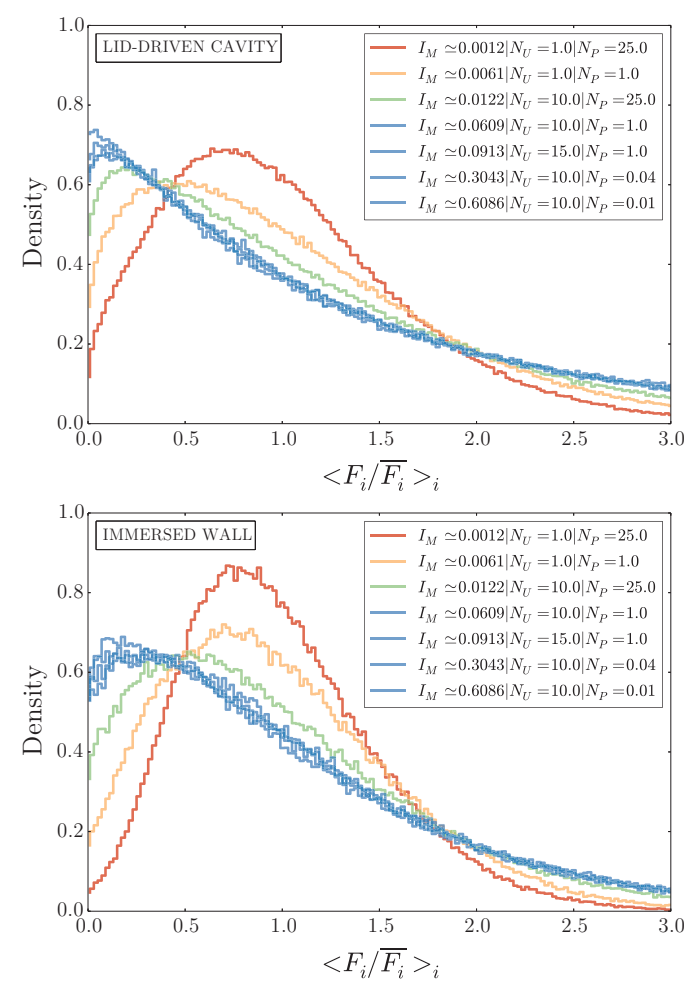

Figure 4. Distributions of the force experienced by a portion of the boundary wall for the lid-driven cavity (top panel, $H$ split into 10 portions) and the immersed wall system (bottom panel, $h$ split into 5 portions). The curves drawn in both graphs correspond to the mean value obtained from all the vertical locations $i$ along the wall, excluding the extreme locations at the top and the foot of the boundary wall

$I_{M}$, as depicted in Fig. 4. The top panel shows the results for the lid-driven cavity, while the bottom panel holds for the immersed wall system.

The force distributions shown in Fig. 4 collapse nicely into one single master curve, independent of $I_{M}$, once $I_{M}$ becomes greater than $10^{-2}$, whatever the granular system considered. This remarkable result, showing that there exists a threshold value of $I_{M}$ which is independent of the system geometry, suggests that the distributions on portions of wall are robust to identify the transition between quasistatic and dense inertial granular flow regimes.

\section{Force time-series autocorrelation}

The autocorrelation function $C(t, t+\Delta t)$ of force timeseries on the wall was analysed for the cavity and the immersed wall system. It was generally observed that $C(t, t+\Delta t)$ dropped quickly (exponentially) to zero over a time scale $\Delta t_{c}$ and could fluctuate around zero (see details in [6, 7]). Note that very similar $C(t, t+\Delta t)$ functions were extracted from an experimental study on the force experienced by an intruder dragged into a granular medium in an annular geometry [8].

Well-defined fluctuations, associated with the transmission of the frequency $U / d$ linked to the roughness of the wall at the top shearing the granular sample, could 


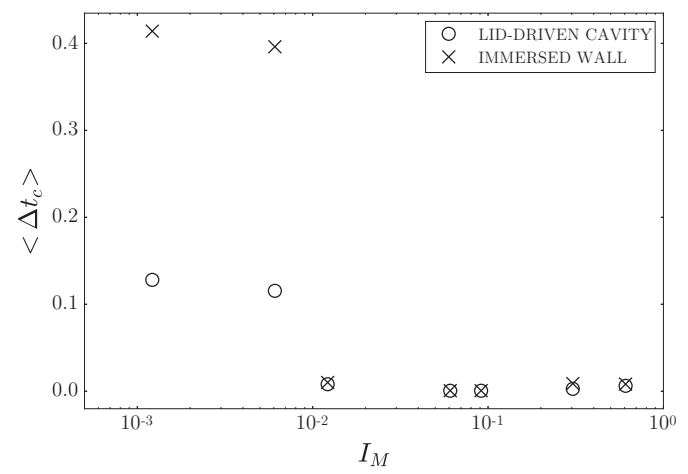

Figure 5. Critical time $\left\langle\Delta t_{c}\right\rangle$ beyond which the force time-series become weakly correlated, as a function of $I_{M}$. We report the mean over the $\Delta t_{c}{ }^{i}$ values for $i \in[1 ; 10]$ in the case of the liddriven cavity (cross symbols) and for $i \in[1 ; 5]$ in the case of the immersed wall system (circle symbols)

sometimes be identified in the case of the cavity, as detailed in [6]. By contrast, this effect was never detected for the immersed wall. This observation illustrates the fact that the cavity can produce high confinement pressure on the right top corner (see [5] for much more discussion).

We could not find any clear relation between the critical time $\Delta t_{c}$ beyond which the force time-series became weakly correlated, and the inertial number $I_{M}$, considering the entire height of the boundary wall subjected to the granular flow. However, by considering the autocorrelation function for portions of wall (as done for the distributions in section 3), we found that each $\Delta t_{c}{ }^{i}$ (the critical time corresponding to a vertical location $i$ along the boundary wall) systematically had a non-zero value for $I_{M}$ below $10^{-2}$, while it was nil beyond $I_{M}=10^{-2}$. This result being robust whatever the system considered, we defined the critical time $\left\langle\Delta t_{c}\right\rangle$ averaged over all the locations $i$ considered along the boundary wall. The latter is plotted in Fig. 5 as a function of $I_{M}$ for the two granular systems. It is remarkable that both curves display very similar behaviours.

The autocorrelation function $C(t, t+\Delta t)$ can be seen as a measure of the memory of the system through time $\Delta t$. High values of $C$ indicate that a future state of the system (in terms of force applied to the wall) depends on the past state of this system, while it does not when $C$ tends toward zero. As such, we can conclude from Fig. 5 that a future state of the system may strongly depend on the past state below $I_{M} \simeq 10^{-2}$ while it does not beyond this critical $I_{M}$. The latter result being robust whatever the granular system considered, this reveals another clear signature of the transition from the quasi-static regime (system with a memory) to the dense inertial regime (full loss of memory).

Note that the non-zero value of $\left\langle\Delta t_{c}\right\rangle$ below $I_{M} \simeq 10^{-2}$ is higher for the immersed wall than for the cavity. This difference may be explained by the fact that the grains against the boundary wall are moving along the cavity wall (perpetual recirculation), while they are not in the immersed wall system because they are trapped in the stag- nant zone formed against the immersed wall. Therefore, memory effects may be more pronounced in the immersed wall system.

\section{Conclusion}

The present paper paid a particular attention to the force fluctuations experienced by the boundary wall, considering a wide range of macroscopic boundary conditions in terms of shearing velocity and confinement pressure imposed to each system. By comparing the results between the two distinct systems, we could extract some key features which appeared to be independent of the system at stake. In particular, our study showed that the distributions measured on portions of wall (of size around $3 d$ ) moved from an exponential decrease at large forces with a shoulder at small forces, which were independent of $I_{M}$ for $I_{M}$ greater than $10^{-2}$, toward $I_{M}$-dependent Gaussianlike distributions while $I_{M}$ was decreased. Moreover, we could detect a system memory for the lowest $I_{M}$, which disappeared while increasing $I_{M}$ beyond $10^{-2}$. Those features were obtained from two distinct granular flow systems, characterized by a different time-averaged dynamics in terms of streamlines and mean force scalings on the boundary wall. Those results then appear as a robust signature of the transition between quasistatic and dense inertial granular flow regimes, revealing and quantifying the link between the $\mu(I)$-rheology proposed by [9] and the force fluctuations experienced by a boundary wall.

\section{Acknowledgements}

This work has been partially supported by the LabEx Tec21 (Investissements d'Avenir: grant agreement No. ANR11-LABX-0030). Thierry Faug and Mohamed Naaim acknowledge the financial support by the People Programme (Marie Curie Actions) of the EU 7th FP under REA Grant Agreement No. 622899 (FP7-PEOPLE-2013IOF, GRAINPACT).

\section{References}

[1] S. J. Antony, Physical Review E 63, 011302 (2000)

[2] F. Radjai, Comptes Rendus Physique 16, 3 (2015)

[3] E. Gardel, E. Seitaridou, K. Facto, E. Keene, K. Hattam, N. Easwar, and N. Menon, Philosophical Transactoions of the Royal Society A 367, 5109-5121 (2009)

[4] https://yade-dem.org/doc/

[5] F. Kneib, T. Faug, F. Dufour, and M. Naaim, Computational Particle Methods 3, 293 (2016)

[6] F. Kneib, T. Faug, F. Dufour, and M. Naaim (submitted manuscript)

[7] F. Kneib, T. Faug, F. Dufour, and M. Naaim (manuscript in preparation)

[8] J. Geng and R. Behringer, Physical Review E 71, 011302 (2005)

[9] P. Jop, O. Pouliquen, Y. Forterre, Nature 441727 (2006) 